\title{
La Biblioteca Virtual de Aldebarán (BVA). Recursos electrónicos gratuitos útiles en un hospital
}

\author{
Por Mercedes Rodríguez del Castillo Martín y Fernando Lechuga Rodríguez del Castillo
}

\begin{abstract}
Resumen: En internet existen numerosos recursos de información de calidad dedicados a las ciencias de la salud y que son de libre acceso. Diferentes instituciones gubernamentales, no gubernamentales y supranacionales ofrecen información con requisitos de rigor científico que pueden resultar de gran utilidad a los profesionales sanitarios. Estos recursos fiables se encuentran en la Red junto a otros con escaso o ningún rigor científico. Los bibliotecarios biomédicos tienen entre sus principales tareas la selección, organización, y análisis de sitios disponibles en la Red para facilitarlos de forma rigurosa, ordenada y útil a sus lectores. En el presente trabajo se describe cómo se ha realizado una biblioteca virtual dirigida a los profesionales sanitarios de un hospital de especialidades, compuesta por recursos de ciencias de la salud de calidad y gratuitos en internet. Se analizan los criterios y objetivos que se han utilizado para la selección y organización de los contenidos.
\end{abstract}

Palabras clave: Internet, Bibliotecas de hospital, Sistemas online, Bibliotecas digitales.

\section{Title: The Aldebarán Virtual Library (BVA). Free electronic resources of use for hospitals}

\begin{abstract}
There are many high quality, health science information resources freely available on the Internet. Many governmental and non-governmental organisations have web pages with very good information on health sciences that are highly useful for health professionals and patients. However, these reliable resources intermingle on the Web with other sites of low quality. The main tasks of today's medical librarians are evaluation, selection, analysis and organisation of resources available on the Web in order to deliver them in a useful form to the end users. This article describes the setting up of the Aldebaran Virtual Library that serves the health staff of a large hospital and consists of high-quality health sciences resources available freely over the Internet. The article focuses on the criteria and objectives used for selecting and organising the content.
\end{abstract}

Keywords: Internet, Hospital libraries, Online systems, Digital libraries.

\section{LAS BIBLIOTECAS DE Como Lindberg y Humphreys} CIENCIAS DE LA SALUD (CS) han sido pioneras a la hora de incorporar tecnologías de la información. Desde la implantación de Medlars en los años setenta, que sirvió como ejemplo a la hora de diseñar sistemas de acceso a la información no sólo en biomedicina, hasta la aparición de internet, las CS han sido de las primeras especialidades científicas que han utilizado las tecnologías más avanzadas del momento para difundir información.

En la actualidad, una de las principales tareas a las que el bibliotecario dedica su tiempo es la selección de buenos recursos electrónicos para sus usuarios, no limitándose a localizarlos, ya que también los organiza de manera que se pueda disponer de ellos fácilmente. (director y subdirectora de la National Library of Medicine, NLM, de los EUA) afirman, "el principal valor de las bibliotecas digitales consiste en las tareas de selección, organización, análisis y ordenación de vínculos realizadas por profesionales expertos ayudados de las tecnologías. En otras palabras, las bibliotecas digitales necesitan todavía a los bibliotecarios".

\section{Objetivos}

La Biblioteca Virtual del Grupo Aldebarán, Gestión del Conocimiento en el Hospital (BVA) se creó con la idea de aprovechar los recursos de información en CS de calidad accesibles de forma gratuita a través de internet. Una vez seleccionados, clasificados y organizados, han sido habilitados en una bi- blioteca virtual para ofrecerlos a los usuarios habituales de la biblioteca general del Hospital Universitario Virgen de las Nieves en particular y, en general, a cualquier profesional sanitario a quien pudiera resultar de utilidad.

http://www.aldebaran.edu.es

El Grupo Aldebarán se fundó para investigar en sistemas y servicios de acceso a la información en CS, y está inventariado dentro del Plan Andaluz de Investigación (PAI) con el número CTS 466.

La $B V A$ está dirigida a tres grupos de usuarios: médicos, enfermeros y pacientes. En la primera fase se ha realizado una recopilación más exhaustiva de los contenidos dirigidos a los médicos y se han incrementado seguidamente los dedicados a enfermeros y a pacientes. 


\section{Dirigida a profesionales sanitarios}

Aparte de los recursos que la biblioteca adquiere, y que nunca resultan suficientes, se considera muy conveniente para sus usuarios aprovechar las posibilidades que existen en internet sobre información en CS de alta calidad y de acceso gratuito.

Pensando en las necesidades de información específicas de los profesionales de un hospital, con la BVA se pretendió tanto recabar los mejores sitios de libre acceso como ordenarlos, clasificarlos y situarlos en una plataforma desde la que se pueda acceder a ellos de la manera más fácil posible, para poder dirigirse a la fuente apropiada según las necesidad de información de cada momento. Además, como valor añadido, se acompaña cada link de una descripción del recurso correspondiente que ayuda a decidir su conveniencia.

\section{Criterios de selección de contenidos}

Se hizo una revisión de la literatura donde se especificaran normas para seleccionar sitios de calidad de información biomédica en la $\operatorname{Red}^{2}$. De igual modo, aparte de los recursos habituales que se utilizan en las bibliotecas biomédicas, se consultaron las bases de datos Pubmed, Bireme, Índice Médico Espanol, Cinalh y Google $e^{3}$.

Se utilizaron los siguientes criterios:

—Utilidad de la información.

—Autoría (fiabilidad).

—Calidad.

—Profundidad de contenido.

-Permanencia y estabilidad del recurso.

—Facilidad de utilización.

-Gratuidad.

—Actualización.

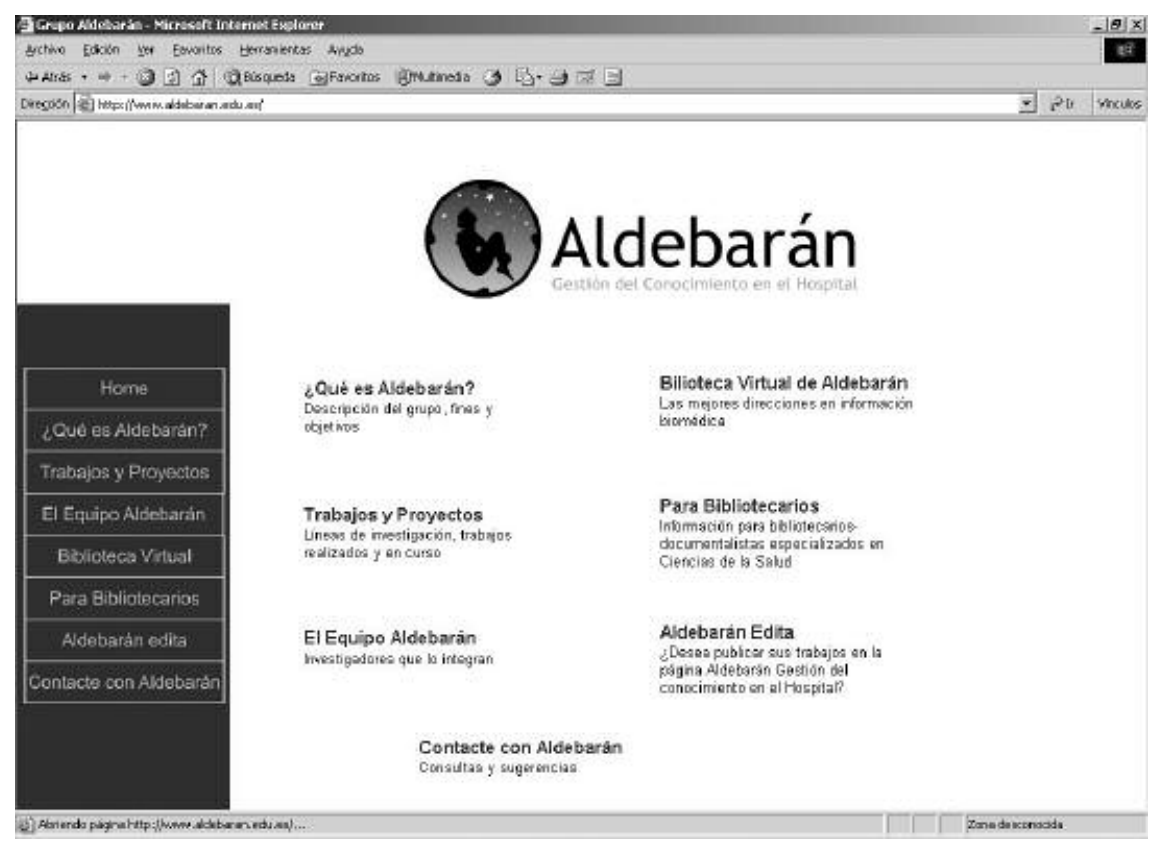

\subsection{Utilidad de la informa-} ción

Los bibliotecarios de hospital suelen conocer bien a sus lectores habituales y las demandas de información de los distintos departamentos que componen la institución. La $B V A$ se basa en las necesidades de información de un gran hospital de especialidades, con 39 servicios y 18 unidades, cuyas tres actividades básicas son la práctica asistencial, la investigación y la docencia. El primer objetivo ha sido recopilar la información de interés para los profesionales que integran estas especialidades y para cubrir las tres actividades básicas.

\subsection{Autoría}

Las páginas elaboradas por algunas de las más prestigiosas organizaciones del mundo no necesitan más aval que ellas mismas. La citada NLM (EUA), el Instituto Karolinska (Suecia), el National Cancer Institute (EUA), el National Health Service (Reino Unido), la Organización Mundial de la Salud, Bireme (Brasil), etc., poseen suficiente prestigio para garantizar su fiabili$\mathrm{dad}^{4}$.

Todas las direcciones seleccionadas para la $B V A$ ostentan visiblemente una autoría bien reconocida, claramente identificada.

\subsection{Calidad}

Existen trabajos rigurosos donde se describen sistemas fiables para seleccionar sitios de calidad especializados en CS y que se han tenido en cuenta en el caso del trabajo llevado a cabo para la $B V A$.

Otro criterio empleado ha sido el patrocinio explícito de las asociaciones médicas más prestigiosas, o las grandes universidades del ámbito internacional, así como la constancia de los sellos de calidad como Hont, por ejemplo 5 .

\subsection{Profundidad de conteni-} do

Según la definición de la American Medical Association (AMA) contenido es: "todo el material (incluido texto, gráficos, cuadros, ecuaciones, audio y vídeo)" ${ }^{\prime}$. Todos los recursos seleccionados por la $B V A$ proporcionan información suficiente y reúnen los requisitos de integridad y actualización. Salvo algún caso excepcional no se acompañan de publicidad.

\subsection{Permanencia}

Muchos usuarios se lamentan de lo efímero de algunas direcciones que han utilizado y la decepción sufrida cuando intentan conectar a ellas de nuevo. Las fuentes recogidas en la $B V A$ son permanentes 
en la Red gracias, en la gran mayoría de los casos, al patrocinio de las importantes instituciones que las sustentan, que a la par que su calidad garantizan su estabilidad.

\subsection{Facilidad de utilización}

Es otra de las características a tener en cuenta aunque, como es natural, en el caso de las bases de datos se necesite un cierto aprendizaje según el grado de complejidad de la búsqueda.

En cualquier caso, como aconseja la $A M A$, cada sitio debe proporcionar un motor de búsqueda o herramienta de navegación apropiada para facilitar su uso.

\subsection{Gratuidad}

Como se ha dicho, en la Red hay muchas fuentes excelentes $\mathrm{y}$ gratuitas. De ellas la más usada es la base de datos Pubmed, de libre acceso a partir de 1997 gracias a la iniciativa de la $N L M$ y del gobierno de EUA.

\subsection{Actualización}

Mantener al día y revisados los elementos que integran la $B V A$ es un punto esencial para obtener el nivel de calidad pretendido.

En realidad todas estas particularidades están relacionadas entre sí y la presencia de una conlleva a menudo las otras. La garantía de calidad está avalada por la autoría, que a su vez sustenta su permanencia, al igual que los medios técnicos que proporciona para facilitar una mejor utilización y también su constante actualización. El hecho de que generalmente se trata de prestigiosas instituciones sin ánimo de lucro financiadas con importantes y seguros presupuestos, hace que se puedan permitir la gratuidad.

\section{Organización de contenidos}

A lo largo de años de experiencia profesional, como decíamos, el bibliotecario de hospital llega a co- nocer bastante bien las necesidades de información de sus usuarios. Sabe lo que necesitan individualmente sus lectores habituales así como lo que requieren de forma general los diferentes servicios que integran la institución. Por supuesto, también está al tanto de las líneas de investigación que se desarrollan y el soporte documental que debe prestar al respecto y, en general, las demandas de información que su institución presenta para el cumplimiento de sus objetivos.

«La BVA está
dirigida a tres
grupos de
usuarios:
médicos,
enfermeros y
pacientes,
siendo el
primero el
colectivo que
más la utiliza»

Conoce también qué tipos de recursos suelen utilizar de forma cotidiana y también los que esporádicamente puedan precisar, el tiempo en que necesitan sus usuarios esta información y de qué modo se les puede facilitar para que resulte más eficaz.

La estructura debe estar dirigida a facilitar la consulta de los distintos especialistas que recaban información para ser aplicada en los cuidados de un enfermo, revisar y actualizar las actuaciones médicas, utilizarla en la investigación que se realiza en el hospital, o como soporte para los planes docentes de la institución.

Se ha seguido por tanto una estructura que coincide con los temas que corresponden a las solicitudes habituales. En orden alfabético:

-Bases de datos.

-Buscadores.

—Estadística.
-Ética.

-Guías de práctica clínica.

-Imágenes.

-Información médica acreditada.

—Investigación.

-Monografías de libre acceso.

-Especialidades médicas.

-Medicina basada en la evidencia.

-Portales.

—Revistas de libre acceso.

\subsection{Bases de datos}

Dado el ingente volumen de publicaciones periódicas dedicadas a las CS, constituyen el elemento básico de consulta de cualquier biblioteca de la especialidad. Desde que a finales de los años ochenta Index Medicus en papel fue sustituido por Medline en cd-rom, hasta que a partir de 1997 Pubmed se hizo accesible gratis en internet -sin olvidar las múltiples versiones online comerciales que datan de principios de los setenta-, las bases de datos de la $N L M$ han sido fundamentales en las bibliotecas biomédicas. También se han tenido presentes por su utilidad otras como: Bireme (bibliografía iberoamericana), las de las Agencias Españolas de Tecnologías Sanitarias, CancerNet, auspiciada por el gobierno de EUA, o la Social Psychology Network. Por otra parte, en los últimos años, los gobiernos en España han decidido subvencionar a través de distintos ministerios el acceso a algunas importantes bases de datos como Cochrane Library y Web of Knowledge, así como a continuar la producción del Índice Médico Español, que indexa la literatura en CS en España, todas las cuales se han incluido en la $B V A$. No se tiene en cuenta Embase porque, a pesar de su indudable importancia, no está disponible de forma gratuita en la Red. 


\section{Versión online de EPI}

Existe una versión electrónica de El profesional de la información, de uso gratuito para todos los suscriptores de la revista, que pueden acceder a través de internet a los textos completos y materiales gráficos publicados desde el año 2000.

Más información en:

http://www.elprofesionaldelainformacion.com/contenidos.html

\subsection{Buscadores}

Se han elegido diferentes herramientas que sólo localizan información en biomedicina aplicando criterios científicos y técnicos. Recogen escritos de carácter académico, artículos revisados, patentes, etc. Es el caso de Scirus, o MedHunt que recuperan información médica de calidad en alrededor de 90.000 documentos; o Trip Database que aplica los principios de la medicina basada en la evidencia (MBE) y recoge información organizándola en categorías como guías de práctica clínica, imágenes, libros, artículos para aplicar a terapia, diagnóstico, etiología, etc. También se ha incorporado Google Scholar.

\subsection{Estadística}

Dentro del ámbito sanitario son frecuentes las búsquedas de información epidemiológica, poblacional, estadística en general, etc.

Se han elegido recursos que tienen datos a nivel nacional como el Instituto Nacional de Estadística, e internacional como el Servicio Británico de Salud con estudios, boletines, publicaciones periódicas, etc. La web de la $O M S$ también se ha tenido en cuenta ya que ofrece su sistema de información estadística.

\section{4. Ética}

Los avances que se están produciendo en biomedicina (ensayos clínicos, manipulación genética y otros) hacen que los profesionales se planteen cuestiones y dudas de conducta y actuaciones en el desarrollo de su actividad, tanto en el trato con los pacientes como en el terreno de la investigación. Aspectos sobre la relación médico-paciente, consentimiento informado antes de ciertas intervenciones, actuaciones congruentes con los conocimientos vigentes, confidencialidad..., preocupan al profesional sanitario y hacen que solicite la información más adecuada al respecto en éstos y otros asuntos de especial naturaleza ${ }^{7,8}$.

De esta forma se han seleccionado las webs de la Biblioteca Ética Kennedy, auspiciada por la $G e$ orgetown University; Ethics in Science and Medicine de la University of Texas at Dallas; The Center for Bioethics de la University of Pennsylvania; etc.

\subsection{Guías de práctica clínica}

Según la definición aceptada, "son recomendaciones desarrolladas sistemáticamente para ayudar a los médicos y a los pacientes a decidir sobre la atención sanitaria más apropiada en circunstancias clínicas concretas". Han adquirido extraordinaria importancia no sólo como herramienta para el clínico sino también para la formación continuada y pre y postgrado. Hay por tanto un gran interés entre los médicos por este tipo de información que ya abunda en la Red'.

\subsection{Imágenes}

Dentro de la medicina existen especialidades que precisan específicamente del uso de imágenes, aparte por supuesto de la importancia que tienen en cualquier actividad docente o en las presentaciones habituales en seminarios, sesiones clínicas y congresos ${ }^{10}$. Por esto se ha dedicado una sección a la recopilación de imágenes procedentes de la NLM, el Instituto Karolinska, The Center for Disease Control and Prevention del Departamento de Salud de EUA. Se recogen ilustraciones, fotografías, animaciones indizadas, etc. ${ }^{11}$.

\subsection{Información médica acre- ditada}

Los continuos avances en biomedicina modifican constantemente los modos de aplicar los cuidados de salud. Este hecho conduce a que médicos e instituciones sanitarias consideren necesario supervisar la calidad de las actividades sanitarias, implementar procesos de evaluación y revisión de las actuaciones, servicios y sistemas sanitarios.

Muchas consultas son sobre estándares y procedimientos sobre la calidad en la práctica asistencial, sistemas de gestión y planificación de salud, servicios de tecnologías sanitarias y cuidados especiales. Se han recogido páginas como la de $U R A C$, organización internacional sin ánimo de lucro, Healthfinder, 
del Departamento de Salud de EUA o Healthinsite patrocinado por el gobierno australiano ${ }^{12}$.

\subsection{Investigación}

El Hospital Virgen de las Nieves cuenta con una Subdirección de Investigación a la que está adscrita la propia biblioteca. Una de sus tareas cotidianas es suministrar información de soporte para esta actividad. Aparte de algunas fuentes dedicadas especialmente a la investigación clínica y a las políticas de salud, también se han incluido recursos cuyo acceso patrocina el gobierno de nuestro país, tal como el ya citado Web of Knowledge $e^{13}$, de ISI, Thomson.

\subsection{Libros}

Se han recogido algunos de los libros más completos y fiables.

\subsection{Especialidades médicas}

La $B V A$ ha recopilado 54 para distribuir por estas materias los intereses de información concretos de los profesionales. En muchos casos se ha recurrido a las páginas que elaboran las más prestigiosas asociaciones de las especialidades respectivas, que a su vez organizan y seleccionan importantes y útiles herramientas dirigidas a los campos específicos de la medicina $^{14}$.

\subsection{Medicina basada en la evidencia}

En la actualidad ha generado vastísimos volúmenes de información y es un tema que suscita innumerables solicitudes de información, por lo que resultaba imprescindible en el proyecto de Grupo Aldebarán. Entre las instituciones que ofrecen en la Red el resultado de sus estudios e investigaciones sobre la MBE están el Servicio Británico de Salud, Cochrane, la NLM y la Universidad McMaster en Canadá ${ }^{15}$.

\subsection{Portales}

Se consideran muy útiles, por lo que se ha habilitado una sección pa-

ra ellos: Fisterra, Infodoctor, The National Electronic Library (Reino Unido), etc.

\subsection{Revistas}

Cada vez son más los accesos a través de la Red de publicaciones periódicas con contenidos revisados por pares. La gran mayoría de ellas son títulos que tradicionalmente se han editado en papel y que ahora cuentan con la edición digital. Un cierto número están accesibles gratis, al menos a partir de una determinada fecha de edición, algunas gracias a iniciativas de origen académico, institucional o científico: Pubmed Central, Scielo, etc. ${ }^{16}$.

\section{«El libre acceso fue considerado esencial para la selección de recursos»}

Hasta aquí se han intentado describir las características, los propósitos y objetivos que llevaron a la creación de la $B V A$. Este proyecto no tendría sentido sin una constante actualización, revisión y mejora del mismo que le conduzca a alcanzar las cotas de calidad y eficiencia que se ha propuesto $y$, por supuesto, con el fin de resultar una herramienta de utilidad para sus usuarios.

\section{Bibliografía}

1. Lindberg, D. A.; Humphreys, B. L. “2015, the future of medical libraries". En: Journal of medicine, 2005, v. 352, n. 11, pp. 1.067-1.070.

\section{Eysenbach, G.; Diepgen, T. L. "Towards} quality management of medical information on the internet: evaluation, labelling, and filtering of information". En: British medical journal, 1998, v. 317, n. 7.171, pp. 1.496-1.500.

3. Gattoni, F.; Sicola, C. "How to evaluate the quality of health related websites". En: Radiology medicine, 2005, v. 109, n. 3, pp. 280-287.

4. Commission of the European Communities, Brussels. "eEurope 2002: quality criteria for health related websites". En: Journal of medical internet research, 2002, v. 4, n. 3, pp. E15.

5. García, S.; Montesinos, E.; Boyer, C. "Iniciativas en español para la evaluación de contenidos biomédicos en internet". Consultado en: 25-07-05. http://www.informaticamedica.org/104/papers/ garcia_70.pdf

6. Winker, M. A.; Flanagin, A.; Chi-Lum, B. White, J.; Andrews, K.; Kennett, R. L.; DeAngelis, C. D.; Musacchio, R. A. "Guidelines for medical and health information sites on the internet: principles governing AMA web sites. American Medical Association". En: Journal of American Medical Association, 2000, v. 283, n. 12, pp. 1.600-1.606.

7. Palmer, K. "Doing the right thing. Hospital ethics committees help clinicians, families, and facilities wrestle with tough questions". En: Minnessota medicine, 2004, v. 87, n. 6, pp. 26-29.

8. Sethuraman, K. R. "Ethics of patient care and trainee-doctors in teaching hospitals". En: Journal of postgraduate medicine, 2003, v. 49, n. 2, pp. 159-162.

9. Field, M. J.; Lohr, K. M. (ed). Institute of Medicine. Guidelines for medical practice. From development to use. Washington D. C.: National Academy Press, 1992.

10. Cook, D. A. "Learning and cognitive styles in web-based learning: theory, evidence and application". En: Academic medicine, 2005, v. 80, n. 3, pp. 266-278.

11. Jastrow, H.; Vollrath L. "Teaching and learning gross anatomy using modern electronic media based on the Visible human project". En: Clinical anatomy, 2003, v. 16, n. 1, pp. 44-54.

12. American Hospital Association. "URAC progressing with web site accreditation". En: Hospital peer review, 2001, v. 26, n. 5, pp. 67-68.

13. Abels, E. G.; Cogdill, K. W.; Zach, L. "Identifying and communicating the contributions of library and information services in hospitals and academic health sciences centers". En: Journal of the Medical Library Association, 2004, v. 92 , n. 1, pp. 46-55.

14. Rambo, N.; Fuller, S. "From bench to bedside: research and testing of internet resources and connections in community hospital libraries". En: Proceedings of the Annual symposium on computer applied medicine care, 1993, pp. 549-553.

15. Hooper-Lane, C.; Combs, A. M.; Feldstein, D. "Finding the best available evidence: what's new?". En: World medical journal, 2005, v. 104 , n. 3 , pp. $8-21$.

16. Wren, J. D. “Open access and openly accessible: a study of scientific publications shared via the internet". En: British medical journal, 2005, v. 330, n. 7.500, p. 1.128 .

\section{Mercedes Rodríguez del Castillo}

Martín, Biblioteca General del Hospital Universitario Virgen de las Nieves. mmerced.rodriguez.sspa@juntadeandalucia.es

Fernando Lechuga Rodríguez del

Castillo, Escuela Superior de Diseño Gráfico, Internet y Multimedia. fernandolechuga82@hotmail.com 